\title{
Mononeuropathy of a distal branch of the femoral nerve in a body building champion
}

\author{
L Padua, E D’Aloya, M LoMonaco, R Padua, B Gregori, P Tonali
}

\begin{abstract}
A unique case of a body building champion with localised atrophy of the distal portion of the vastus lateralis muscle is reported; neurophysiological evaluation suggests a selective lesion of a distal branch of the vastus lateralis nerve (a motor branch of the femoral nerve). A necroscopic study in four cases was performed to better clarify the site and mechanism of nerve lesion. The data suggest that stretching and compression of the nerve has probably occurred during strenous exercise.
\end{abstract}

(F Neurol Neurosurg Psychiatry 1997;63:669-671)
Keywords: femoral nerve; mononeuropathy; body building; anatomical study

Femoral mononeuropathy is uncommon. ${ }^{1-4}$ Usually the lesion is located in the proximal tract, involving all muscles innervated by the femoral nerve. One case with an idiopathic isolated femoral mononeuropathy affecting the whole vastus lateralis has been recently described, ${ }^{5}$ but no cases with partial atrophy of the vastus lateralis have been reported.

We report an atypical case of femoral mononeuropathy. To identify the putative site of lesion an anatomical necroscopic study was performed in four cases.
Istituto di Neurologia L Padua

M LoMonaco

B Gregori

P Tonali

Istituto di Medicina

Legale

E D'Aloya

Istituto di Ortopedia, Università Cattolica SC, Roma, Italy R Padua

CSS Hospital IRCCS S Giovanni Rotondo, Italy

P Tonali

Correspondence to: Dr Luca Padua, Institute of Neurology, Università Cattolica,

Lgo F Vito 1 00168, Rome, Italy.

Received 20 February 1997 and in revised form 19 May 1997

Accepted 30 May 1997

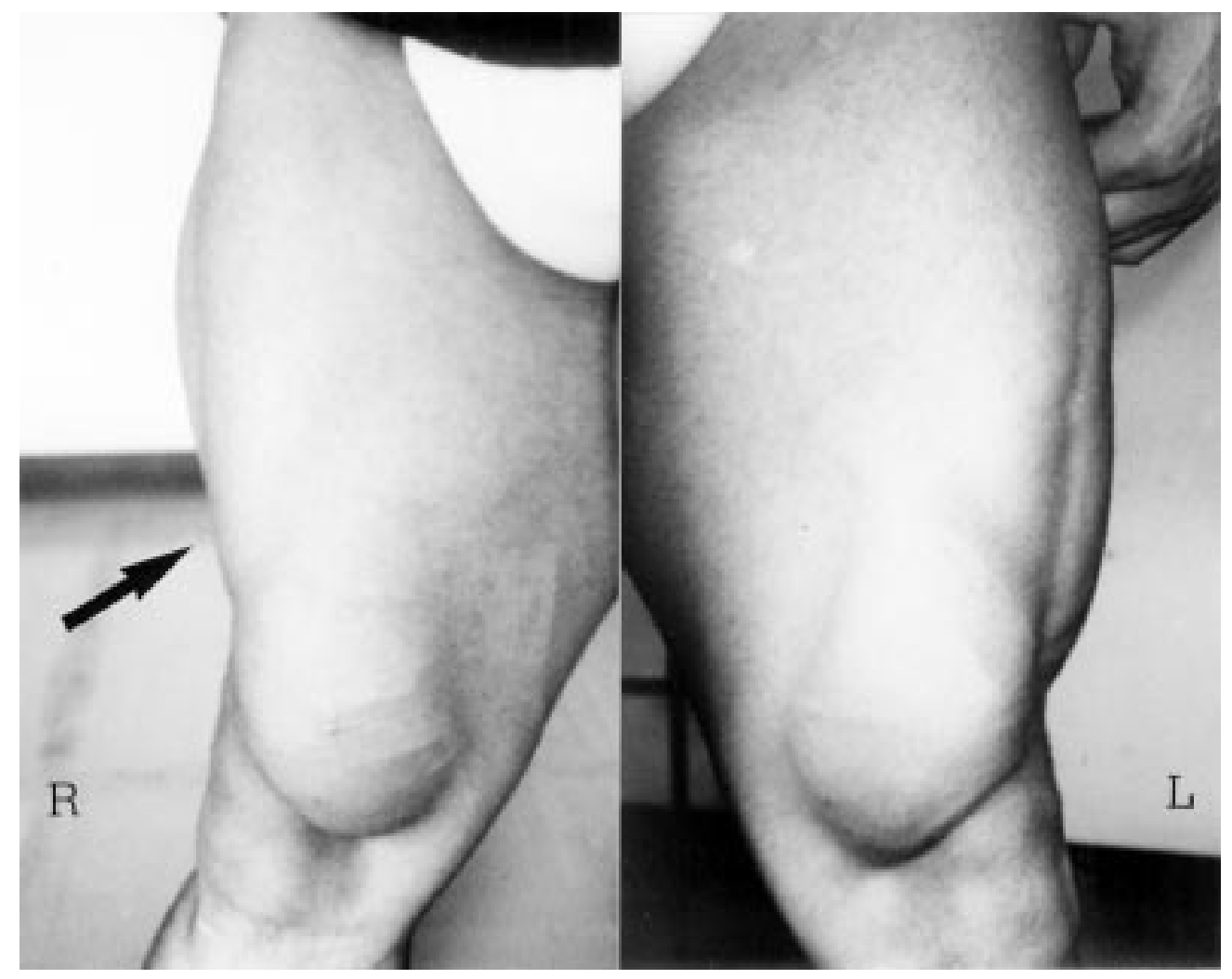

Figure 1 Atrophy of the distal portion of the vastus lateralis muscle (see arrow) on the right side of a body building champion; on the left side the vastus lateralis muscle was normal. 

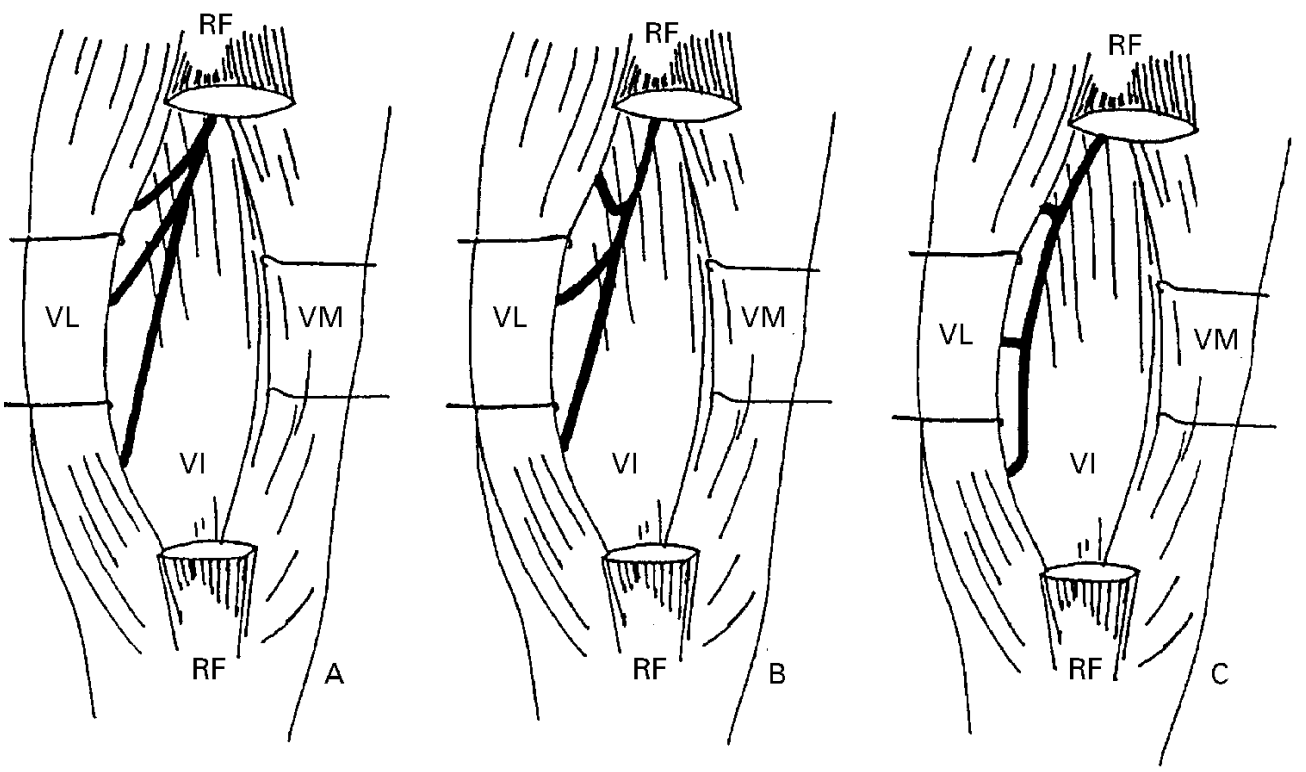

Figure $2(A, B, C)$ Patterns of the anatomical region of the vastus lateralis motor branches as they appeared in the anatomical study. The pattern of innervation in (B) was found in two dissections. $R F=$ rectus femoris tendon; VL=vastus lateralis; VM=vastus medialis; VI=vastus intermedius.

\section{Case report}

A 32 year old male body building champion noted, one month before examination, atrophy of a distal region of his right thigh. He did not complain of back or leg pain. No trauma was referred. Neurological examination showed an atrophy of the distal third of the vastus lateralis (figure 1) with a diminished right patellar reflex; no motor or sensory deficit was present.

Needle EMG of the distal portion of vastus lateralis showed positive sharp waves and fibrillation potentials at rest. The patient was not able to evoke voluntary activity in the atrophic portion of the muscle; moreover, no motor response (needle electrode) from this portion of muscle was detected after stimulation of the femoral nerve at the groin. An EMG of the right medium and proximal portion of the vastus lateralis muscle was normal (with normal femoral nerve conduction). EMG findings of the iliopsoas, rectus femoris, vastus medialis, and tibialis anterior were normal. Results from nerve conduction studies of the right median, peroneal, and tibial nerves and bilateral sensory nerve conduction studies of the saphenous and sural nerves were normal. Echography of the right inguinal and thigh regions showed no pathological mass. Creatine kinase and serial fasting blood sugars were within normal limits.

It was recommended that he temporarily suspend the sports activity but the patient could not comply with this suggestion because of competition. Ten months later, the muscle atrophy and neurophysiological evaluation were unchanged.

ANATOMICAL AND NECROSCOPIC STUDY

The femoral nerve (branch of the lumbar plexus) divides, in the femoral triangle, into its terminal branches, one of which is directed towards the posterior side of the vastus latera- lis. The motor branch directed to this muscle subdivides to give two to four terminal branches (for the proximal, median, and distal portion of the muscle). ${ }^{67}$ These branches penetrate from the back forward through the vastus lateralis muscular mass and innervate it.

To better identify the course of the nerve to the vastus lateralis, we performed four necroscopic dissections in four subjects. These anatomical studies showed a uniform path of the motor branch of the vastus lateralis muscle along the space defined by the fasciae and muscular masses of the quadriceps femoris. The posterior surface is represented by the superior face of the vastus intermedius, the anterior limit is constituted by the posterior face of the rectus femori, and the external and internal walls are represented by the margins of the vastus medialis and vastus lateralis.

Slight between subject differences in the origin of the vastus lateralis nerve from the femoral nerve and in the angle existing between the vastus lateralis nerve and its terminal branches were detected. We noted, in all four cases, three terminal motor branches supplying the vastus lateralis, which arise at different levels and perforate from the muscular mass with either a right angle or an acute angle (figure 2).

\section{Discussion}

In our patient, clinical data (painless, isolated atrophy, no systemic disease, exercise stress) and neurophysiological findings (isolated complete denervation, no abnormal sign in the other muscles innervated by the same roots or nerve, normal findings at the saphenous nerve) strongly suggest an isolated mononeuropathy of the motor branch innervating the distal portion of the right vastus lateralis.

Concerning the mechanism of nerve injury, we think, as do other authors, that nerve stretch plays a key part in damage to the femoral nerve, 
especially in athletes and in patients with "hanging leg syndrome". ${ }^{13-5}$ The hypothesis of the stretch mechanism is strengthened by the fact that body builders develop the capability of contracting distinct muscles of their quadriceps femoris in isolation. In our patient, this might result in a "scissoring" phenomenon. Moreover, we suggest that in our patient nerve compression also occurs at the sliding plane between the hypertrophied muscles during excessive muscle contraction.
1 Rottemberg MF, DeLisa JA. Severe femoral neuropathy with "hanging leg" syndrome. Arch Phys Rehabil 1981;62:404-6.

2 Hakim MA, Katirji MB. Femoral mononeuropathy induced by lithotomy position: a report of 5 cases with a review of literature. Muscle Nerve 1993;16:891-5.

3 Sammarco GJ, Stephens MM. Neuroapraxia of the femoral nerve in a modern dancer. Am 7 Sport Med 1991;19:413-4.

4 Miller EH, Benedict FE. Stretch of the femoral nerve in a dancer. F Bone Foint Surg Am 1985;67:315-7.

5 Carter GT, McDonald GM, Chan TT, Margherita AJ. Isolated femoral mononeuropathy to the vastus lateralis: EMG and MRI findings. Muscle Nerve 1995;18:341-4.

6 Pensa A. Trattato di anatomia umana. Torino: Utet, 1978

7 Testut L. Traité d'anatomie humaine. Paris: G Doin, 1898. 